\title{
Host diet influences parasite diversity: a case study looking at tapeworm diversity among sharks
}

\author{
Trent K. Rasmussen ${ }^{1, *}$, Haseeb S. Randhawa ${ }^{1,2,3,4}$ \\ ${ }^{1}$ Ecology Degree Programme, University of Otago, PO Box 56, Dunedin 9054, New Zealand \\ ${ }^{2}$ Present address: Falkland Islands Fisheries Department, Directorate of Natural Resources, Falkland Islands Government, \\ Bypass Road, Stanley FIQQ 1ZZ, Falkland Islands \\ ${ }^{3}$ Present address: South Atlantic Environmental Research Institute, PO Box 609, Stanley Cottage, Stanley FIQQ 1ZZ, \\ Falkland Islands \\ ${ }^{4}$ Present address: New Brunswick Museum, 277 Douglas Avenue, Saint John, New Brunswick E2K 1E5, Canada
}

\begin{abstract}
In theory, animal diets may act as important filters for different parasite species. However, there is currently a lack of empirical research looking at host diet as a potential predictor of parasite diversity among different host species. The aim of this study was to assess the influence of several host diet features (including diet breadth, diet composition, and trophic level) on tapeworm diversity in sharks, relative to other key factors, including host size, habitat, phylogeny, latitude, and depth. Data on these host features were compiled from a comprehensive analysis of literature records including 91 different shark species, and 3 measures of tapeworm diversity were examined: tapeworm species richness, tapeworm taxonomic distinctness (TD), and variance in tapeworm TD. The diet breadth of a shark species was revealed to be a better predictor of tapeworm species richness than other host features examined to date. Host size, trophic level, diet TD, latitudinal range, and the mid-point of a shark's depth range also significantly influenced tapeworm richness when analyses were adjusted to prevent confounding by phylogenetic relationships between hosts. The TD of tapeworm assemblages was influenced by diet breadth, diet TD, host size, and depth range when analysed independently of host phylogeny. Overall, our findings demonstrate that aspects of host diet have important consequences for parasite diversity in sharks. We emphasise that studies of parasite diversity in other systems should more seriously consider including aspects of host diet (particularly diet breadth) as potential key predictors of parasite diversity.
\end{abstract}

${ }^{*}$ Corresponding author: t.k.rasmussen@outlook.com

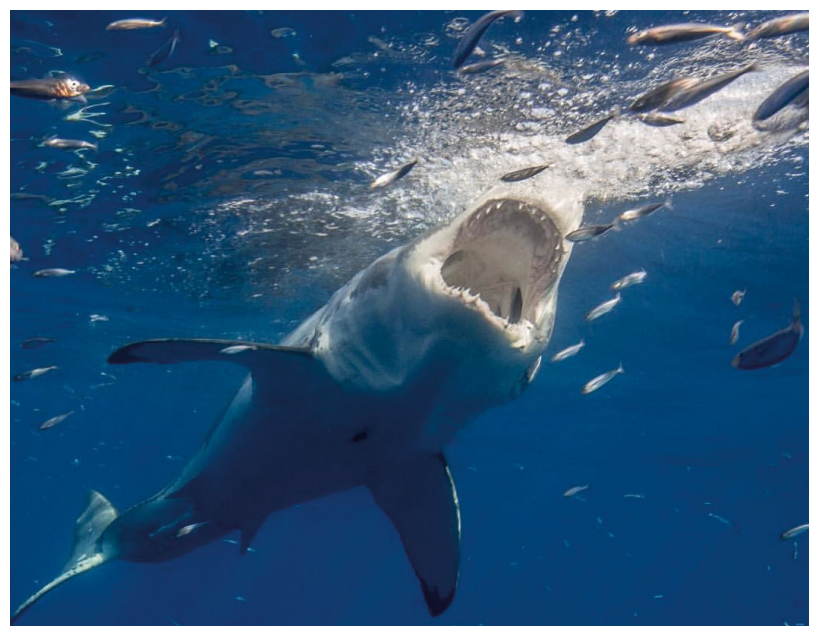

The variety of prey in sharks' diets may have a large influence on how many parasite species infect them.

Photo: Martin Graf

KEY WORDS: Sharks - Cestodes - Tapeworms · Species richness $\cdot$ Diet $\cdot$ Species diversity $\cdot$ Taxonomic distinctness $\cdot$ Phylogenetically independent contrasts

\section{INTRODUCTION}

The diversity of parasite species in a host, like the diversity of free-living species in an ecosystem, is shaped by a range of different ecological and evolu-

() The authors 2018. Open Access under Creative Commons by Attribution Licence. Use, distribution and reproduction are unrestricted. Authors and original publication must be credited. 
tionary features. Exploring how these features relate to parasite diversity is fundamental to our understanding of why particular host species evolve with richer or more diverse parasite faunas than others (Poulin 2004). Research over the past several decades has already identified several widely important host features that can influence the diversity of parasite assemblages, including body size, lifespan, population density, geographical range, and diet (e.g. Morand et al. 2000, Vitone et al. 2004, see also Kamiya et al. 2014). A few of these features, such as body size, population density, and geographical range, have been recognised as 'universal' determinants of parasite species richness (Kamiya et al. 2014). Despite the general significance of these few host attributes, however, their relative importance varies considerably among different host-parasite systems (e.g. Poulin et al. 2011), and depending on which hosts and parasites are involved, other less generalised factors may also have a large influence on parasite diversity. For instance, in anthropoid primates, parasite species richness is influenced largely by social group size (Vitone et al. 2004). In addition, a number of host features, which could potentially have a significant impact on parasite diversity, are often left out of studies investigating determinants of parasite diversity. For example, host diet and metabolic rate have seldom been included in comparative analyses, making it difficult to assess the predictive strength of these factors (Kamiya et al. 2014). In light of these details, there remains a need for research to assess the relative importance of different host features in various host-parasite systems, to better understand emerging diseases and their transmission dynamics.

Sharks and their tapeworm species assemblages provide a useful system for looking at large-scale patterns of parasite diversity for a number of reasons. First, the tapeworm parasites of these elasmobranchs show substantial diversity and exhibit high host specificity. They are the most diverse group of parasites infecting elasmobranchs (Caira \& Healy 2004), with 1034 different species and 202 genera known from 9 established orders (Caira et al. 2017). Based on the number of host species sampled for parasites to date, it is also estimated that ca. 3600 tapeworm species in described elasmobranchs have yet to be recorded (Randhawa \& Poulin 2010). Almost all of these tapeworm species exhibit a high degree of host specificity in sharks, with most being restricted to a single host species or a few closely related hosts (Caira \& Jensen 2014). However, it is also worth noting that these tapeworms are generally less specific in intermediate hosts (Palm \& Caira 2008), and can have consequences for thousands of marine species other than elasmobranchs, including a broad range of teleost fishes, molluscs, crustaceans, mammals, reptiles, and other invertebrates (Jensen 2009). Second, sharks are a group of animals that have features with broad and easily measurable variation across species. Shark species display a broad range of sizes, are found in nearly all marine habitats, depths, and latitudes, and show marked variation in the range of their depth and latitude distributions (Froese \& Pauly 2017). There is also considerable variation in diet and trophic level among different species (Cortés 1999). Third and finally, sharks are key apex predators of marine ecosystems and, along with their many parasites, they exert considerable influences throughout marine food-webs. Thus, sharks and their parasites represent an important model for understanding determinants of marine parasite diversity.

From previous research on tapeworm diversity in sharks, several host features, including latitude, depth, and habitat, appear to have little influence on their parasite diversity (Randhawa \& Poulin 2010). Host size has been identified as a significant predictor, but no other ecological or host biological characteristics examined to date have demonstrated significant effects on shark tapeworm diversity (Randhawa \& Poulin 2010). Additionally, shared host evolutionary history, i.e. shark phylogeny, outweighs ecological variables as a predictor of tapeworm diversity in sharks (Poulin et al. 2011). However, a number of potentially important host features have yet to be examined for their influence on tapeworm diversity. Host population density is an attribute of likely importance, but is not feasible for investigation in sharks due to data deficiencies. Of the factors for which data are available, aspects of host diet are arguably the most prominent features warranting investigation. Host diet has not previously been assessed as a determinant of shark tapeworm diversity, but it is likely to have a large impact considering that all tapeworm species in elasmobranchs are acquired via ingestion of infected prey (which are intermediate or paratenic hosts) (Williams \& Jones 1994). Essentially, the number of tapeworm species that infect a shark species should directly depend on which prey, and ultimately how many different prey, a shark consumes as part of its regular diet.

The main aim of this study was to examine whether 3 major aspects of host diet, namely (1) breadth of diet, (2) trophic level, and (3) diet composition, influence the species diversity of tapeworm assemblages in sharks. Although all 3 of these factors convey 
information on the diet of sharks, each is distinctly different in what it tells us. Breadth of diet is a general measure of how many different prey are consumed by a shark species. We hypothesised that shark species with broader diets (diets including a greater diversity of prey taxa) would harbour a greater diversity of tapeworm species than those with restricted diets. This was based on the fact that sharks with broad diets would encounter more tapeworm intermediate hosts through their diet, and thus, may come in contact with a greater diversity of larval parasites. Trophic level is a measure of a species' position in food webs, and indicates overall what type of ecological group is most important in the diet (see Cortés 1999). We hypothesised that shark species occupying higher trophic levels would have more diverse tapeworm assemblages than those occupying lower trophic levels, since they have access to more trophic links and additional intermediate hosts from higher trophic levels. Diet composition is a measure of which specific taxa are the most dominant in the diet of a host species. In contrast to trophic level, which reflects the position of a shark's prey in the food web, diet composition tells us which specific taxonomic groups of prey (e.g. teleosts, cephalopods, crustaceans) comprise most of the diet. We predicted that shark species feeding predominantly on teleost fishes would have more tapeworm species than other groups because most currently described intermediate hosts for marine tapeworms are teleost fishes (Palm 2004, Jensen 2009). Overall, we predicted that each of these measures of host diet would have more significant, higher-magnitude impacts on tapeworm diversity than host features examined in previous studies.

\section{MATERIALS AND METHODS}

\section{Tapeworm data collection}

All tapeworm species diversity data used in this study were compiled by revising and updating a comprehensive elasmobranch tapeworm dataset made available by Randhawa \& Poulin (2010). This original data set obtained tapeworm richness estimates for a large range of shark species by searching through the 'Zoological Records' on ISI Web of Knowledge and compiling available data from 1864 to 2008 . To ensure that our data set was accurate and up to date, we revised the original data set by changing tapeworm richness estimates to include all new sharktapeworm records published in the 'Zoological Re- cords' from 2008 to 2017. Also, a number of shark species were included in our data set for which tapeworm species records had become available since 2008. Using the same method as Randhawa \& Poulin (2010), new tapeworm records for each host species were found by searching the shark taxa (binomials plus all known synonyms) combined with the key words 'parasit* OR disease OR pathog*'. Notably, the presence of a tapeworm species in the spiral valve of a shark host can sometimes involve immature worms or represent a temporary infection that does not allow the parasite to complete its life cycle. To ensure that our tapeworm species diversity data were as reliable as possible, we only included infection records which specified that tapeworms were present as adults in the spiral valve. Moreover, when the development stage or site of infection was unavailable for a tapeworm species, we did not include the record in our dataset.

Since measures of parasite diversity are often greatly influenced by sampling/study effort (Walther et al. 1995), correcting for sampling effort can give a more accurate measure of diversity (Poulin 2004, Luque \& Poulin 2007). An ideal measure of parasite diversity sampling effort would be the sum of host individuals examined for parasites, but unfortunately, these data are seldom available from studies describing new parasite records. As such, we measured the sampling effort for parasite diversity of each host species as the total number of references obtained by searching the host's tapeworm records on ISI Web of Knowledge (using the search parameters defined above). Compared with other estimates of sampling effort, this measure has been previously demonstrated as a stronger correlate with tapeworm richness (Randhawa \& Poulin 2010).

To complement tapeworm species richness as a measure of parasite diversity, we also calculated the average taxonomic distinctness (TD) of tapeworm assemblages for each shark species. This index is used to measure the average taxonomic distance between the parasite species of an assemblage, with greater TD values indicating greater average taxonomic difference between species in the assemblage (Luque et al. 2004). TD is a different measure of diversity to richness in that it is thought to be more sensitive to host ecology (Luque et al. 2004, Luque \& Poulin 2008). It is measured as the average number of steps up the taxonomic hierarchy (Phylum, Class, Order, Family, Genus, and Species) in order to reach a taxonomic level common to 2 species, and is calculated for all pairs of species in the assemblage being examined (Warwick 
\& Clarke 2001). We computed variance in TD to accompany the measure of TD for each shark species wherever possible. Variance in TD is not as useful as the TD index itself, but can be used to provide information on the taxonomic heterogeneity among host species, basically showing how even the distribution of taxa across the taxonomic tree is (Warwick \& Clarke 2001). We calculated the tapeworm TD and the associated variance for each host species harbouring at least 3 tapeworm species, using the program 'Taxobiodiv 1.2' (available at www.otago.ac.nz/parasitegroup/downloads.html).

\section{Host features data collection}

For all shark species with an available estimate of tapeworm richness, we recorded a number of important host features: (1) diet breadth, measured as the total number of prey families in a shark's diet; (2) diet $\mathrm{TD}$, measured as the average taxonomic distance between all prey families in a shark's diet; (3) trophic level, measured as the number of energy-transfer steps to the shark's food chain position (estimated from the relative contribution of different prey groups to the diet) (Cortés 1999); (4) diet composition, given as the taxonomic prey category composing most of the diet (of 9 groups: teleost fishes, cephalopods, crustaceans, mammals, chondrichthyan fishes, reptiles, birds, other molluscs, other invertebrates); (5) habitat, given by the categorical summary of the species' habitat (of 7 groups: reef-associated, demersal, pelagicoceanic, pelagic-neritic, benthopelagic, bathypelagic, bathydemersal); (6) host total length, measured as the maximum recorded length for a species; (7) latitudinal range, measured by the number of degrees of latitude spanning the shark's geographic distribu-

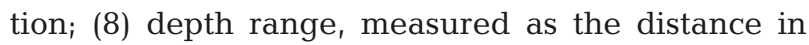
metres between the shallowest and deepest points at which the shark occurs; (9) depth mid-point, measured as the mid-point of the shark's preferred depth distribution; and (10) phylogeny, represented by the Genus, Family, and Order of the host. It is worth noting that many of these host characteristics (length, latitudinal range, depth range, depth mid-point, and habitat) have been previously assessed as predictors of tapeworm diversity (Randhawa \& Poulin 2010), and were also included in the present data set to determine their relative importance compared with the dietary features of primary interest here.

Data on most host features (phylogeny, trophic level, length, latitudinal range, depth range, depth mid-point, and habitat) were obtained directly from recent species records listed on FishBase in March 2017 (Froese \& Pauly 2017). In cases where data on one or more of these host features were not available for a species, the shark species was excluded from the data set. For all data on host diet, we compiled a comprehensive dataset from ISI Web of Knowledge. To do this, we searched the taxon of each shark (binomial plus all known synonyms) combined with the key words 'diet* OR feed* OR prey*' on ISI Web of Knowledge (all databases) and amassed all available references from 1864 to 2017 (searches were conducted in April 2017). Every reference listed was perused for information on the diet of sharks, and out of 2081 references listed across all species, 361 had relevant data on diet that could be included in this study (see Table S1 in the Supplement at www.intres.com/articles/suppl/m605p001_supp.pdf).

Unsurprisingly, the level of taxonomic definition for prey varied among studies, where some sharks had prey recorded mostly to Family or Order level, others had prey items known to the level of Species. Considering this potential bias in records, it was decided that the best measure of diet breadth would be at the taxonomic level of Family. At this level, there were records for most species that had tapeworm diversity estimates, and there was also a large range of taxonomic groups (603 different families of prey) to give a good measure of variability in diet breadth among sharks. To ensure further accuracy in diet breadth comparisons, we also restricted the final data set to only include host species with at least 1 family of prey recorded $(\mathrm{n}=91)$. From each reference, we recorded every family of prey identified for each shark species. Notably, some diet records were old, and taxonomic changes had been made to certain prey taxa since their publication. To address this, we checked all prey taxa in the World Register of Marine Species (WoRMS) database and updated prey families to their current taxonomical nomenclature (WoRMS Editorial Board 2017).

Similarly to measures of parasite diversity, measures of diet breadth may be substantially affected by sampling effort (Randall \& Myers 2001). Diet breadth is likely to increase as more individual hosts are examined for diet contents. Correcting for this influence of sampling effort should therefore provide a better measure of host diet (Cortés 1999). In contrast to studies describing parasites from sharks, the standard protocol for dietary studies is to include the number of hosts examined. Thus, we were able to measure diet sampling effort as the sum of stomachs containing food (i.e. the number of non-empty stomachs) examined across all diet records for each shark species. For 
a few references, the number of non-empty stomachs examined was not provided, and therefore had to be excluded from further analyses. All references were checked thoroughly in the methods and results sections to make sure that diet information was not duplicated among studies. In cases where studies gave duplicate data, the data were cited from the original reference only, and the number of hosts examined was only included for the original reference to avoid overestimation of sampling efforts.

TD of the diet was calculated to complement the number of prey families as a measure of diet breadth. Similarly to the TD calculations for tapeworm assemblages, TD of prey family assemblages and associated variance were computed for each shark species with at least 3 families of prey in their diet, using 'Taxobiodiv 1.2'.

For information on diet composition, we recorded the percentage composition of 9 different prey groups from each reference (teleost fishes, cephalopods, crustaceans, mammals, chondrichthyan fishes, reptiles, birds, other molluscs, other invertebrates). The overall composition of these groups for each shark species was then calculated by taking the average compositions across all studies, weighted by the number of non-empty stomachs examined for diet. Similar to the approach of Cortés (1999), we used compound indices to estimate composition where available (e.g. the index of relative importance, $\%$ IRI), and otherwise, single indices, such as percent frequency of occurrence $(\% \mathrm{O})$, percent number $(\% \mathrm{~N})$ percent weight $(\% \mathrm{~W})$, or percent volume $(\% \mathrm{~V})$ were used individually. Where 2 of these single indices were available, an average was calculated (e.g. \%O $+\% \mathrm{~W} / 2$ ). Plant materials, detritus, and non-organic materials were not included in composition estimates, as we were only interested in prey which are potential intermediate hosts for shark tapeworms.

\section{Data analysis}

Statistical tests were carried out in the R environment (R Development Core Team 2012). Prior to analysing relationships between measures of tapeworm diversity and recorded host features, regressions were run to determine the influence of sampling effort on measures of tapeworm diversity and diet breadth. As expected, for both tapeworm richness and diet breadth, the relationships between diversity and sampling effort were best characterized by positive curves where diversity increased with increasing sampling effort, but with the increase slow- ing towards an asymptote at higher effort values. To determine the significance of the associations, we ran simple quadratic regressions of each diversity measure on their associated measures of sampling effort. Host-parasite sampling effort had a significant influence on tapeworm species richness $\left(\mathrm{r}^{2}=0.539\right.$, $\mathrm{p}<$ $0.001)$, but not on tapeworm $\operatorname{TD}\left(\mathrm{r}^{2}=0.007, \mathrm{p}=\right.$ $0.825)$. To correct for this influence in further analyses, tapeworm richness was from here on measured by the residuals of its quadratic regression on hostparasite sampling effort. Diet breadth was also influenced by sampling effort; the number of stomachs sampled for diet showed a strongly positive association with number of recorded prey families $\left(\mathrm{r}^{2}=\right.$ $0.601, \mathrm{p}<0.001)$, although not with prey family TD $\left(r^{2}=0.047, p=0.272\right)$. Thus, there was a need to correct diet breadth, but not diet TD, in further analyses. From this point forward, diet breadth (prey family richness) was measured by the residuals of its quadratic regression on diet sampling effort.

We used linear mixed-effects models (LMMs) to analyse relationships between measures of tapeworm diversity and all recorded host features, each with identity link functions and Gaussian error distribution. Our first main LMM looked at the influence of host features on tapeworm richness (response variable). This model included 7 continuous (fixed) predictor variables (diet breadth, diet TD, trophic level, host length, depth mid-point, depth range, and latitudinal range) and 3 categorical (random) predictor variables (habitat type, dominant diet group, and host phylogeny [measured as host genus nested within host family, nested within host order]). Our second main LMM looked at tapeworm TD as a response with the same predictors as the above model. To supplement the analysis of tapeworm TD, we also ran a model where tapeworm TD and prey TD variables were replaced with associated variances in TD. This was done to gauge the taxonomic 'evenness' among host species. In addition to these LMMs, we conducted a 'tips' analysis, which involved re-running each model with the random effects removed. This analysis serves as an approach to examining differences to the importance of predictor variables when the phylogenetic relationships among hosts are not considered.

The LMMs were analysed in detail using the 'MuMIn' package in R (Barton 2013). Akaike's information criterion corrected for small sample size $(\mathrm{AICc})$ and Akaike model-averaged weights $(w+(i))$ were calculated for all possible linear regression models (models with all possible combinations of the predictors), and used to determine the best models as well as the rank and relative importance of each indi- 
vidual predictor in each model. Model-averaged parameter estimates and 95\% confidence intervals were also calculated for each variable using methods summarised by Anderson (2008), and partial ${ }^{2}$ values for each averaged parameter were assessed using the 'r2glmm' package in R (Jaeger 2016). To determine the interaction terms to be included in each model, we selected a priori sets of second-order interactions between predictor variables that were thought to be relevant based on biological and ecological principles. For instance, there is a known association between host size and trophic level that should be accounted for (Poulin \& Leung 2011). We compared AICc values between models including these sets and the models with only combinations of individual predictors to determine whether inclusion of the interaction terms significantly improved the models. All other potential interactions between predictors were assessed in the same way to make sure that no important interactions were missed. From these analyses, it was decided that 4 interaction terms were to be included in the model predicting tapeworm richness (habitat and depth mid-point, diet breadth and diet TD, diet breadth and trophic level, trophic level and host size), and 3 were to be included in the model predicting tapeworm TD (diet breadth and diet TD, host size and trophic level, latitudinal range and depth range).

Due to the large number of parameters in each LMM compared with the shark sample size $(\mathrm{n}=91)$, it was necessary to check models for over-fitting. Over-fitting was tested by re-running the analyses including (1) only the top 4 predictor variables identified by the highest AIC relative model weights (diet breadth, diet TD, trophic level, latitudinal range, plus their interactions and all random effects), and (2) only the top 4 predictor variables identified by highest partial $\mathrm{r}^{2}$ values (diet breadth, diet TD, latitudinal range, depth range, plus their interactions and all random effects). These 2 tests showed identical outcomes to the main LMMs (and their associated tips analyses), with the relative importance and significance of predictors remaining the same in both. Based on these outcomes, we decided that LMM analyses would continue with all predictors of interest included.

\section{Phylogenetically independent contrast analysis methods}

Analyses of tapeworm richness and TD were repeated using the phylogenetically independent con- trast (PIC) method (Felsenstein 1985) to control for confounding effects of shared evolutionary ancestry on shark features used in these analyses. Contrasts were computed using the PDAP: PDTREE program (Midford et al. 2011) implemented in Mesquite version 3.31 for Mac OSX (Maddison \& Maddison 2017). We derived contrasts from a tree generated using an alignment consisting of previously published molecular data for the NADH dehydrogenase subunit 2 (NADH2) (Fig. 1) for 85 shark species, and 3 outgroups consisting of 1034 sites. Sequences were aligned using MacClade 4.07 (Maddison \& Maddison 2005). The automatic model selection (Beta version) - Smart Model Selection (Lefort et al. 2017) - function in PhyML 3.0 (Guindon et al. 2010) determined the best nucleotide-substitution model for the data. A general time reversible (GTR) model with proportion of invariant sites (I) and gammadistributed rate heterogeneity $(\mathrm{G})$ provided the best fit to the data based on the AICc. The dataset was analysed by maximum likelihood (ML) and Bayesian inference (BI), which were performed using PhyML 3.0 and MrBayes v3.2 (Ronquist et al. 2011), respectively. ML analyses were performed using 6 substitution rate categories, with $\mathrm{G}$ and I set at 0.689 and 0.319, respectively (estimated from Smart Model Selection; very similar to those reported by Randhawa et al. 2015 using jModelTest 0.1.1; Guindon \& Gascuel 2003, Posada 2008), tree improvement set at subtree pruning and regrafting (SPR), and 1000 bootstrap replicates. BI was performed using the covarion option according to a GTR + I + G nucleotide substitution model with no initial values assigned and with empirical nucleotide frequencies. Four separate Markov chains were used to estimate posterior probabilities over $25 \times 10^{6}$ generations, sampling the Markov chains at intervals of 100 generations. The first 50000 trees were discarded as 'burn-in', and then a $50 \%$ majority-rule tree was constructed from the subsequent trees. Nodal support was estimated as the mean posterior probabilities (Huelsenbeck et al. 2001) using the 'sumt' command. The trees were rooted on 3 outgroup taxa, Chimaera monstrosa, C. phantasma, and Hydrolagus collei (see Naylor et al. 2012).

Prior to performing PIC analysis, we calculated phylogenetic inertia (Pagel's lambda) using the package Geiger (Harmon et al. 2008) in the program R (R Development Core Team 2012) and determined that there was a strong phylogenetic signal. We proceeded to compute contrasts following guidelines suggested by Garland et al. (1992). PICs were not run for variance in tapeworm TD, since the dataset 


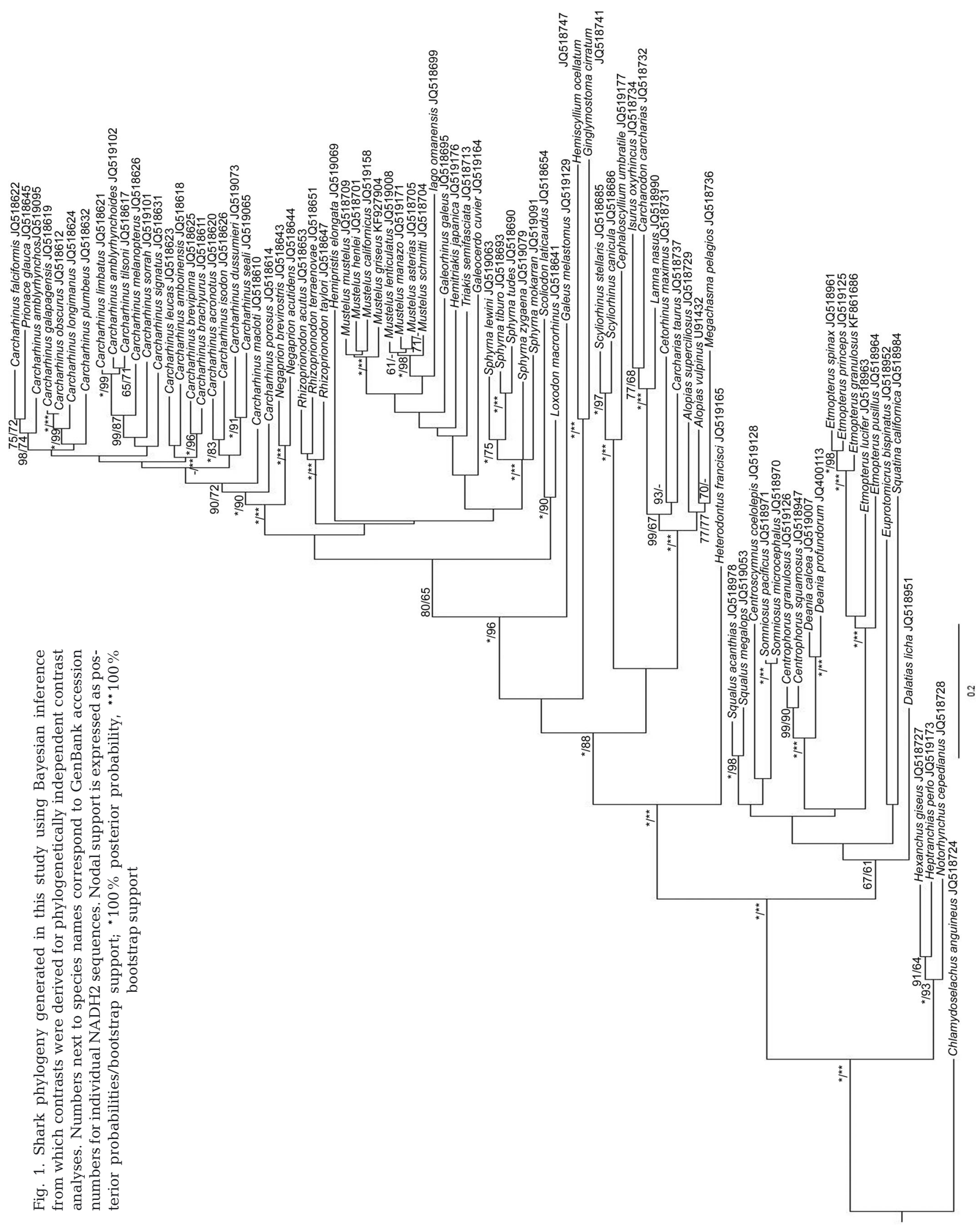


for this response variable was considerably more restricted ( $\mathrm{n}=57$ species), and the LMM results for variance in tapeworm TD did not warrant further investigation.

\section{RESULTS}

Across the 91 shark species analysed in the present data set, we identified a total of 570 tapeworm-host associations. Based on the raw data, shark species harboured 6.26 tapeworm species on average $( \pm 6.00 \mathrm{SD}$, range $=1$ to 24 ; Fig. 2), and the average TD and variance in TD of tapeworm assemblages was $3.39 \pm 0.39$ (range $=1.90$ to 4.17 ) and $0.74 \pm 0.51$ (range $=0.00$ to 2.53 ) hierarchical steps, respectively. Host species were commonly infected by a single tapeworm species, and there was a positive skew in the number of tapeworm species infecting sharks (frequency of shark species decreased with increasing tapeworm richness; Fig. 2). In total, the host-parasite study effort across the 91 shark species included 2068 records, which equated to $22.73 \pm$ 35.67 records host ${ }^{-1}$.

A total of 603 families of prey (within 163 orders, 39 classes, 16 phyla) were recorded in the data set. Shark species had records, on average, of $39.49 \pm 34.16$ families of prey (range $=$ 1 to 145 ; Fig. 3), and the average TD and variance in TD of prey family assemblages was $2.81 \pm 0.56$ (range $=$ 0 to 4.00 ) and $1.12 \pm 0.42$ (range $=0$ to 2.05) hierarchical steps, respectively. As illustrated in Fig. 3, diet breadth (prey family richness), similarly to tapeworm richness, was distributed with a positive skew across the shark species examined. In total, the number of stomachs examined across all shark species exceeded 170000 , with 110005 stomachs containing food. Notably, the number of stomachs with food examined was highly variable among species $($ mean $=1208.85 \pm 4502.72$ ), and the spiny dogfish Squalus acanthias alone had data from 40698 stomachs containing food. The average trophic level of sharks in this dataset was $4.14 \pm$ 0.34 (3.20 to 4.94$)$.

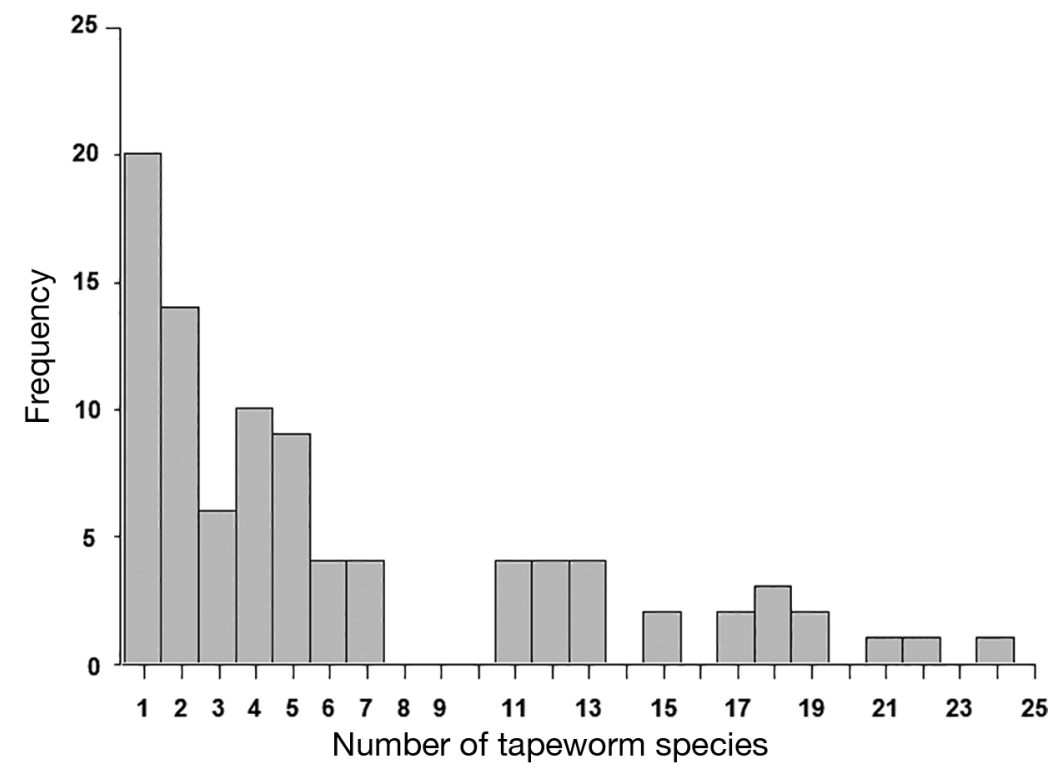

Fig. 2. Frequency distribution of the number of shark species $(n=91)$ infected with different numbers of tapeworm species

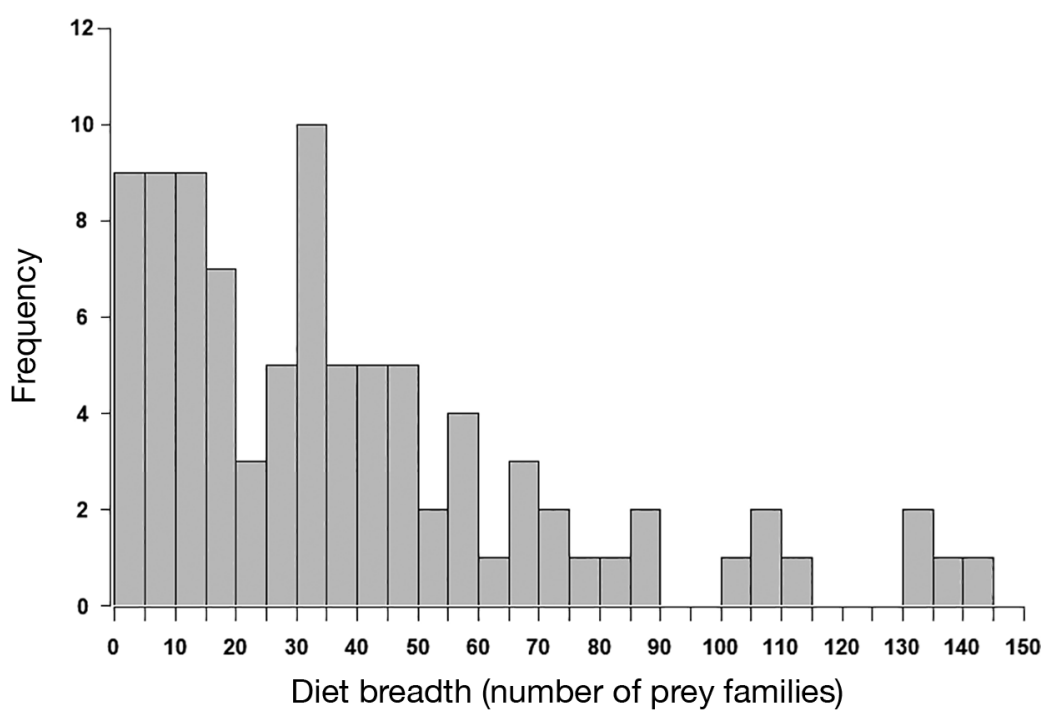

Fig. 3. Frequency distribution of the number of shark species $(n=91)$ consuming various quantities of prey families

\section{Determinants of tapeworm richness}

Our LMM analysis of tapeworm richness revealed that random effects explained a total of $19.1 \%$ of observed variation in tapeworm richness, with the interaction of habitat and depth mid-point explaining $13.26 \%$ and host phylogeny explaining $5.84 \%$, but habitat and diet composition each explaining a negligible proportion of the overall variation (Table 1). The top AIC model explaining variation in shark tapeworm richness (AICc $=508.59$ ) included a com- 
bination of only 3 fixed predictors: diet breadth, diet $\mathrm{TD}$, and trophic level. The top 5 best AICc models were very close $(\triangle \mathrm{AICC}<1)$, and included various combinations of diet breadth, diet TD, trophic level, and the interaction effect of diet breadth and diet TD. The multi-model average approach produced an averaged model that explains $62 \%$ of the total variance in tapeworm richness. The model-averaged Akaike weights analysis also showed that diet-related variables were the best predictors of tapeworm richness. The factor with the highest relative variable weight was diet breadth $(w+(i)=0.99)$, followed by diet TD $(0.71)$, trophic level $(0.59)$, and the interaction between diet breadth and diet TD (0.37) (Table 1). It was interesting to note that although the association between diet breadth and tapeworm richness was positive (tapeworm richness increased with increasing diet breadth), the association between diet TD and tapeworm richness was negative (diet TD decreased with increasing tapeworm richness; Table 1). Most other variables, including latitudinal range, depth mid-point, depth range, and host size, were generally poor predictors of tapeworm richness in comparison (all $w+(i) \leq 0.01)$. As Table 1 shows, 3 predictors had significant effects on tapeworm richness, namely the interaction between diet breadth and diet TD, the interaction between diet breadth and trophic level, and depth range (their 95\% confidence intervals excluded ' 0 '). However, it should be noted that the effect size for depth range was small and only marginally significant $(95 \%$ CI $=[-0.002,-0.000], p=$ 0.044 ; Table 1). The relationship between diet breadth and tapeworm richness was further analysed to determine the strength of the association. A plotted linear regression (Fig. 4) shows a significant positive correlation between the variables with a moderate amount of variability surrounding the linear trend line $\left(\mathrm{r}^{2}=0.220, \mathrm{p}<0.001\right)$. Significant positive correlations were also found when analysing this relationship without the dataset's 2 most dominant (speciose) shark genera (excluding Carcharhinus spp., $\mathrm{r}^{2}=0.201, \mathrm{p}<0.001$; excluding Mustelus spp., $\mathrm{r}^{2}=0.199, \mathrm{p}<0.001$; excluding both, $\mathrm{r}^{2}=0.181$, $\mathrm{p}=0.001$; Fig. 4).

Table 1. Summary of host features as predictors for tapeworm species richness in sharks. Relative importance of predictors is compared by model-averaged weight $(w+(i))$, rank, parameter estimates, $95 \%$ confidence interval $(\mathrm{CI})$, and partial $\mathrm{r}^{2}$ values. Bold: statistically significant $(\mathrm{p}<0.05)$ effects. TD: taxonomic distinctness

\begin{tabular}{|c|c|c|c|c|c|}
\hline \multicolumn{2}{|l|}{ Random effects } & \multicolumn{2}{|c|}{$\begin{array}{c}\text { Number of } \\
\text { levels }\end{array}$} & \multicolumn{2}{|c|}{$\begin{array}{c}\text { Variance } \\
\text { explained (\%) }\end{array}$} \\
\hline Host phylogeny (Genus/Fam & iily/Orde & ler) & 40 & 5.84 & \\
\hline Habitat & & & 7 & $<0.01$ & \\
\hline Diet composition & & & 6 & $<0.01$ & \\
\hline Habitat $\times$ Depth mid-point & & & 81 & 13.26 & \\
\hline Fixed effects & $w+(i)$ & Rank & Estimate & $95 \% \mathrm{CI}$ & $r^{2}$ \\
\hline Diet breadth & 0.99 & 1 & 0.288 & $-0.332,0.909$ & 0.136 \\
\hline Diet TD & 0.71 & 2 & -2.047 & $-4.705,0.611$ & 0.096 \\
\hline Trophic level & 0.59 & 3 & 0.806 & $-1.758,3.369$ & 0.001 \\
\hline Diet breadth $\times$ Diet TD & 0.37 & 4 & -0.124 & $-0.213,-0.037$ & 0.075 \\
\hline Diet breadth $\times$ Trophic level & 0.14 & 5 & -0.115 & $-0.211,-0.019$ & 0.068 \\
\hline Latitudinal range & 0.01 & 6 & 0.005 & $-0.020,0.031$ & 0.009 \\
\hline Depth range & $<0.01$ & 7 & -0.001 & $-0.002,-0.000$ & 0.038 \\
\hline Host size & $<0.01$ & 8 & -0.003 & $-0.007,0.002$ & 0.000 \\
\hline Depth mid-point & $<0.01$ & 9 & -0.001 & $-0.004,0.001$ & 0.004 \\
\hline Host size $\times$ Trophic level & $<0.01$ & 10 & 0.000 & $-0.000,0.000$ & 0.000 \\
\hline
\end{tabular}

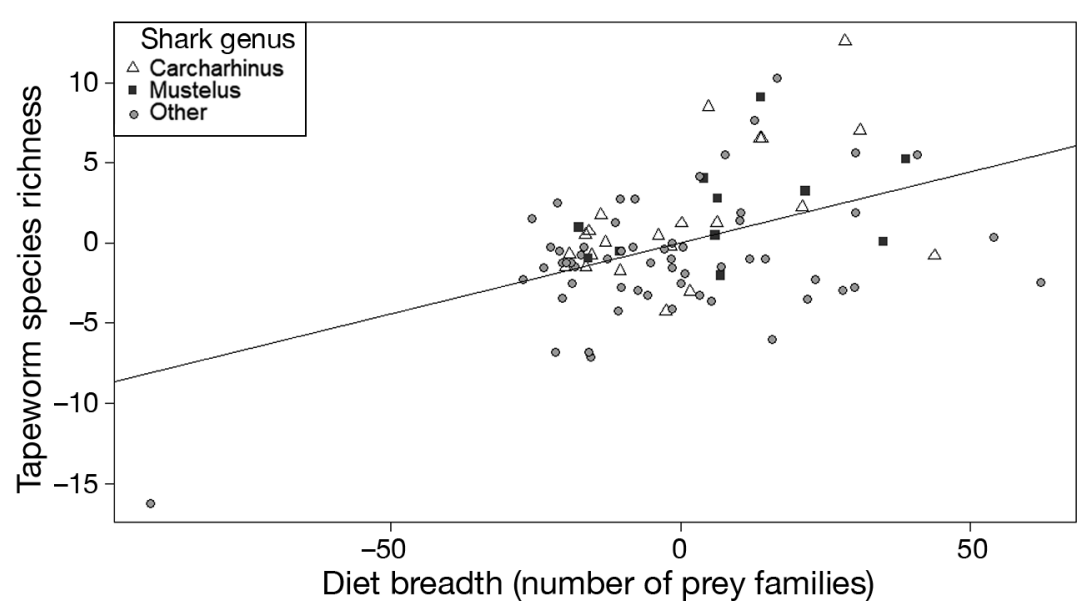

Fig. 4. Association between tapeworm richness (measured as the residuals for the quadratic regression of the number of tapeworm species on parasite sampling effort) and shark diet breadth (measured as the residuals for the quadratic regression of number of prey families on prey sampling effort). Legend indicates which points belong to the 2 most dominant shark genera in the dataset (Carcharhinus spp. and Mustelus spp.). Solid line represents overall linear regression line between the variables $\left(r^{2}=0.220, p<0.001\right)$ 
Table 2. Summary of phylogenetically independent contrasts (PIC) analysing the significance of various shark host features (based on $\mathrm{r}^{2}, t, F$ and p-values) for 2 measures of their tapeworm diversity: species richness and taxonomic distinctness (TD). Bold: statistically significant $\left({ }^{*} \mathrm{p}<0.05\right.$, $\left.{ }^{* *} \mathrm{p}<0.01,{ }^{* * *} \mathrm{p}<0.001\right)$

\begin{tabular}{|c|c|c|c|c|c|}
\hline & $\mathrm{r}^{2}$ & $t$ & $F$ & df & $\mathrm{p}$ \\
\hline \multicolumn{6}{|c|}{ Tapeworm species richness } \\
\hline Tapeworm TD & 0.389 & 7.27 & 52.82 & 83 & $<0.0001^{* * *}$ \\
\hline Diet breadth & 0.529 & 9.66 & 93.34 & 83 & $<0.0001^{* * *}$ \\
\hline Diet TD & 0.366 & 6.93 & 48.00 & 83 & $<0.0001^{* * *}$ \\
\hline Variance in diet TD & 0.289 & 5.77 & 33.32 & 83 & $<0.0001^{* * *}$ \\
\hline Trophic level & 0.055 & 2.19 & 4.79 & 83 & $\mathbf{0 . 0 3 1}^{*}$ \\
\hline Latitudinal range & 0.102 & 3.07 & 9.45 & 83 & $\mathbf{0 . 0 0 3}^{*}$ \\
\hline Depth range & 0.010 & 0.91 & 0.83 & 83 & 0.365 \\
\hline Depth mid-point & 0.047 & 2.02 & 4.06 & 83 & $0.047^{*}$ \\
\hline Host size & 0.092 & 2.90 & 8.42 & 83 & $0.005^{* *}$ \\
\hline \multicolumn{6}{|l|}{ Tapeworm TD } \\
\hline Diet breadth & 0.515 & 9.40 & 88.28 & 83 & $<0.0001^{* * *}$ \\
\hline Diet TD & 0.295 & 5.90 & 34.81 & 83 & $<0.0001^{* * *}$ \\
\hline Variance in diet TD & 0.200 & 4.52 & 20.47 & 83 & $<0.0001^{* * *}$ \\
\hline Trophic level & 0.004 & -0.54 & 0.29 & 83 & 0.590 \\
\hline Latitudinal range & 0.000 & -0.10 & 0.01 & 83 & 0.924 \\
\hline Depth range & 0.116 & -3.30 & 10.92 & 83 & $0.0014^{* *}$ \\
\hline Depth mid-point & 0.037 & -1.78 & 3.18 & 83 & 0.078 \\
\hline Host size & 0.238 & 5.09 & 25.89 & 83 & $<0.0001^{* * *}$ \\
\hline
\end{tabular}

When the analysis was repeated with phylogeny and the other random effects removed, diet breadth, diet TD, the interaction between diet breadth and diet TD, and the interaction between diet breadth and trophic level all became highly significant predictors of tapeworm richness ( $p<0.001$, Table S2). In contrast, depth range became non-significant ( $\mathrm{p}=$ 0.276 , Table S2). These predictors similarly became significant when using the PIC method to adjust for the effects of host phylogeny, and in fact, when adjusted for phylogeny using PICs, all predictor variables had statistically significant effects on tapeworm richness except for depth range (Table 2). The effects of diet breadth and diet TD when adjusting for phylogeny were particularly significant, both explaining large amounts of variation in tapeworm species richness (diet breadth: $t=9.66, \mathrm{r}^{2}=0.529, \mathrm{p}<0.0001$, Fig. 5a); diet TD: $t=6.93, \mathrm{r}^{2}=0.366, \mathrm{p}<0.0001$, Fig. 5b). Interestingly, unlike in the other analyses of tapeworm richness, diet TD showed a positive association with tapeworm richness in the PIC analysis.

\section{Determinants of tapeworm TD}

Our LMM analysis of predictors of tapeworm TD showed that random effects overall accounted for $26.91 \%$ of the variation in tapeworm TD, and host phy- logeny explained a much larger proportion of variation in tapeworm TD $(20.15 \%)$ than it did of tapeworm richness (Table 3). The top AIC model explaining variation in tapeworm TD $(\mathrm{AICC}=71.75)$ was the null model (model including none of the fixed predictors in the data set). Notably, the null model was considerably better than all other AIC models (for all others, $\triangle \mathrm{AICC}$ $>2$ ). Diet TD and trophic level were the best predictors of tapeworm TD included in the model, with the highest relative variable weights across all models (diet TD $[w+(i)=0.23]$ and trophic level [0.10]). However, these model weights were still notably low, and ultimately, all of the variables included in the analysis were poor predictors of tapeworm TD. In addition, no variables demonstrated statistical significance for tapeworm TD (Table 3). When the analysis was repeated with phylogeny and the other random effects removed, latitudinal range $(w+(i)=0.45)$ and depth range $(0.41)$ became the best predictors of tapeworm TD (Table S2). However, all variables remained poor predictors of tapeworm TD overall, and none of the host features included demonstrated significance in this analysis (Table S2). Conversely, most of the predictors demonstrated significant effects on tapeworm TD when using the PIC method to account for phylogenetic relationships (Table 2). Similarly with the PIC analysis for tapeworm richness, when PICs were used to adjust for the effects of host phylogenetic relationships on tapeworm TD, diet breadth $\left(t=9.40, \mathrm{r}^{2}=0.515, \mathrm{p}<0.0001\right.$, Fig. 5e), diet TD ( $t=5.90, \mathrm{r}^{2}=0.295, \mathrm{p}<0.0001$, Fig. 5f), and host size $\left(t=5.09, \mathrm{r}^{2}=0.238, \mathrm{p}<0.0001\right.$, Fig. 5h) all showed significantly positive associations with tapeworm TD (Table 2). In contrast, trophic level, depth mid-point, and latitude were non-significant for tapeworm TD, while depth range showed a significant effect on tapeworm TD (Table 2).

Our model looking at determinants of variance in tapeworm TD (results available in Table S3) was similar to the LMM for TD, with the null model being favoured. A large portion of the variance (58.64\%) was explained by host phylogeny (no other random effects explained variance), but all fixed variables were non-significant and poor predictors of variance in TD. When the analysis was repeated with random effects removed, all factors remained poor predictors of variance in tapeworm TD (see Table S2).

\section{DISCUSSION}

The main objective of this study was to determine which host features influence the diversity of tapeworm assemblages in sharks, with particular focus on 

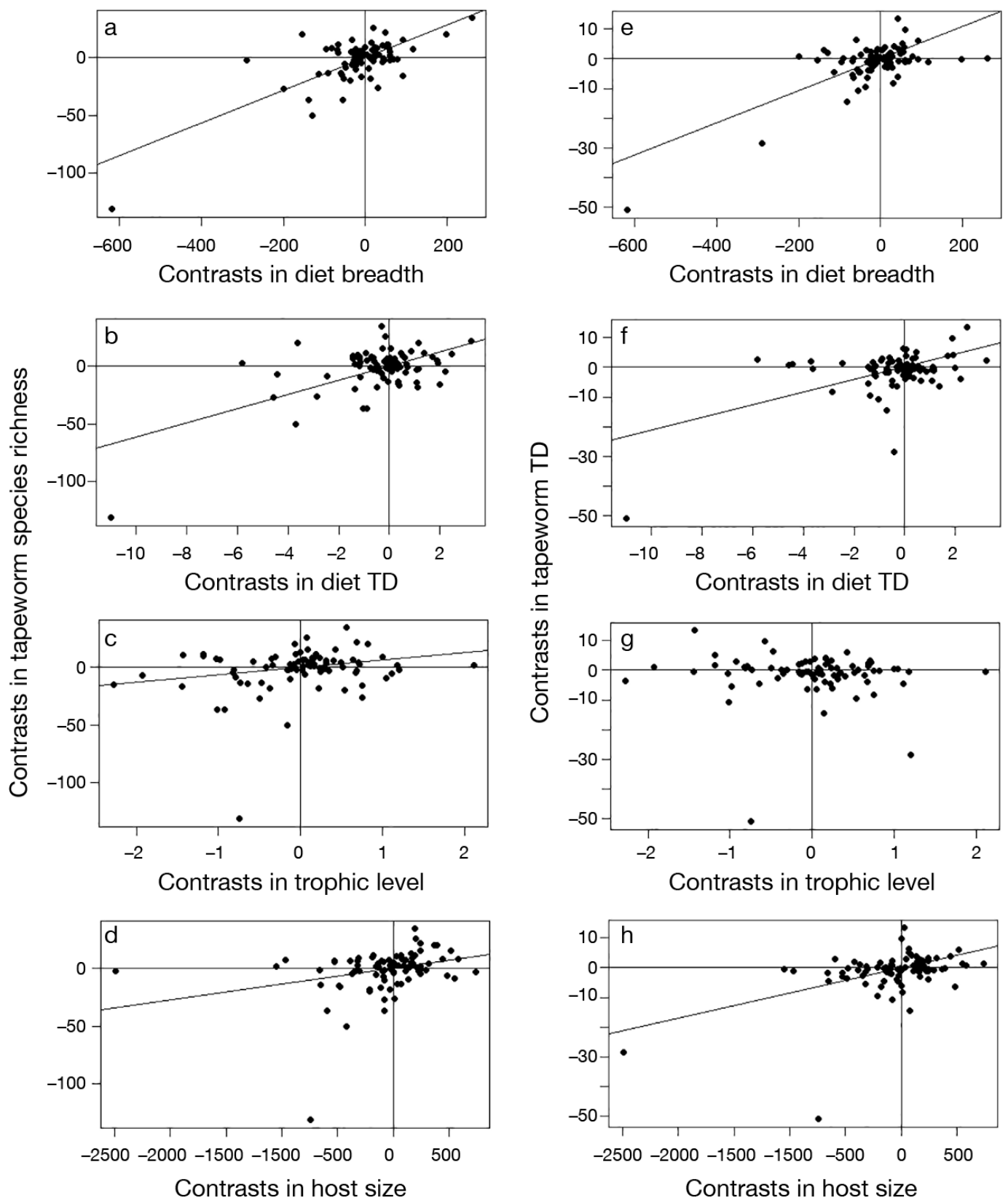

Fig. 5. Relationships between tapeworm species richness (corrected for study effort) and the (a) diet breadth (b) diet taxonomic distinctness (TD), (c) trophic level, and (d) host size of shark species. Relationships between TD of tapeworm assemblages (corrected for study effort) and the (e) diet breadth, (f) diet TD, (g) trophic level, and (h) host size of shark species. Each plot is based on 84 phylogenetically independent contrasts. Solid lines are shown for significant trends and indicate best fit lines for simple linear regressions

the influence of certain aspects of host diet, such as diet breadth, composition, and trophic level. In accordance with this objective, we found that the breadth of a shark's diet, measured by the diversity of prey families, was a better predictor of tapeworm richness than any other host feature examined to date (Table 1). This outcome was robust, with both para- site richness and diet breadth corrected to prevent confounding by their associated sampling efforts, and remained significant even with the exclusion of dominant taxa (Carcharhinus spp. and Mustelus spp.) from the dataset. Moreover, diet breadth showed a highly significant positive association with tapeworm richness after adjusting data to account for phyloge- 
Table 3. Summary of various host features as predictors for tapeworm species taxonomic distinctness (TD) in sharks. Relative importance of these predictors is compared by model-averaged weight $(w+(i))$, rank, parameter estimate, $95 \%$ confidence interval $(\mathrm{CI})$ and partial $\mathrm{r}^{2}$ values

\begin{tabular}{|c|c|c|c|c|c|}
\hline \multicolumn{2}{|l|}{ Random effects } & \multicolumn{2}{|c|}{$\begin{array}{c}\text { Number of } \\
\text { levels }\end{array}$} & \multicolumn{2}{|c|}{$\begin{array}{c}\text { Variance } \\
\text { explained (\%) }\end{array}$} \\
\hline Host phylogeny (Genus/F & iily/Ord & & 29 & 20.15 & \\
\hline Habitat & & & 6 & 2.25 & \\
\hline Diet composition & & & 5 & 4.51 & \\
\hline Fixed effects & $w+(i)$ & Rank & Estimate & $95 \% \mathrm{CI}$ & $\mathrm{r}^{2}$ \\
\hline Diet TD & 0.23 & 1 & -0.210 & $-0.447,0.027$ & 0.037 \\
\hline Trophic level & 0.10 & 2 & 0.012 & $-0.331,0.356$ & 0.011 \\
\hline Diet breadth & $<0.01$ & 3 & 0.001 & $-0.004,0.005$ & 0.001 \\
\hline Latitudinal range & $<0.01$ & 4 & -0.001 & $-0.004,0.002$ & 0.045 \\
\hline Host size & $<0.01$ & 5 & 0.000 & $-0.001,-0.001$ & 0.033 \\
\hline Depth mid-point & $<0.01$ & 6 & 0.000 & $-0.000,0.001$ & 0.040 \\
\hline Depth range & $<0.01$ & 7 & -0.000 & $-0.000,0.000$ & 0.080 \\
\hline Diet breadth $\times$ Diet TD & 0.00 & 8 & 0.000 & $-0.000,0.000$ & 0.001 \\
\hline Host size $\times$ Trophic level & 0.00 & 8 & 0.000 & $-0.000,0.000$ & 0.036 \\
\hline $\begin{array}{l}\text { Latitudinal range } \times \\
\text { Depth range }\end{array}$ & 0.00 & 8 & 0.000 & $-0.000,0.000$ & 0.052 \\
\hline
\end{tabular}

netic relationships between shark species (Table 2, Fig. 5a). Thus, the findings here support the hypothesis that shark species with broader diets encounter and subsequently acquire more tapeworm species than those with restricted diets. So far only a few empirical studies in other host-parasite systems have shown diet breadth to be important for the diversity of trophically transmitted parasites (e.g. Chen et al. 2008, Locke et al. 2014). However, it is also worth noting that other studies looking at determinants of parasite diversity have rarely included diet breadth, and in fact, too few comparative studies have involved diet for meta-analysis to sufficiently assess its strength as a predictor of parasite richness (Kamiya et al. 2014). In such cases, there is an eminent need for more research into the diet of hosts. Where diet records are available, on the other hand, we suggest that future studies involving trophically transmitted parasites should consider finding ways to analyse diet breadth as a potential predictor of parasite richness among host species.

Using the average TD of species assemblages to complement simpler measures of species diversity (i.e. species richness) has been a common practice in ecological studies for the past couple of decades (Von Euler \& Svensson 2001, Heino et al. 2005, Winter et al. 2013). But despite its potential application as a measure of diversity in species diets, to our knowledge TD has only been implemented as a measure of diet breadth in 1 recent study involving the diet of turtles (Stringell et al. 2016). In the present study, we found that diet TD (represented by the TD among prey families in the diet) was the secondbest predictor of tapeworm species richness in sharks following diet breadth (prey family richness), and similar to general diet breadth, diet TD showed a highly significant positive effect on tapeworm richness when PICs were used to control for confounding of phylogenetic relationships among sharks. Interestingly, in the LMM of tapeworm richness, diet TD displayed a negative estimate, entailing a net decrease in tapeworm richness with increasing diet TD. However, when PICs were used to control for the confounding of host phylogeny, diet TD showed a highly significant positive association with tapeworm richness (Table 2, Fig. 5b). This discrepancy suggests that the relationship between tapeworm richness and diet TD may be confounded by host relationships, and emphasises the importance of controlling comparative analyses of host traits for phylogenetic influences. This point is even further reinforced by the fact that most of the host features included as predictors of tapeworm richness showed major differences in significance when the analysis was adjusted for the host's phylogenetic relationships.

The discrepancies described above between our LMM and PIC analyses might relate to the strong bias in species included in our analyses towards the shark order Carcharhiniformes. Although Carcharhiniformes is indeed the most speciose shark order (see Naylor et al. 2012, Randhawa et al. 2015), the diet composition and parasite communities in sharks of this order seem to be positively disproportionally sampled, described, and defined than those of other orders such as the Orectolobiformes or Squaliformes (Table S1). This bias is reflected in the species included in our phylogeny (Fig. 1) and has left many gaps in different families such as the Orectolobidae (Order Orectolobiformes), Scyliorhinidae (Order Carcharhiniformes), and Squalidae (Order Squaliformes). PIC analyses are more sensitive to phylogeny due to the assumptions of accurate branch lengths and correct topology (Felsenstein 1985, Ackerly 2000), hence any taxon bias or phylogenetic uncertainty, e.g. polytomies, might lead to questionable results that must be interpreted carefully (Ack- 
erly \& Reich 1999). Our phylogenetic analyses clearly yielded a flawed topology (see Naylor et al. 2012). For instance, they failed to resolve the Scyliorhinidae (Scyliorhinus canicula, S. stellaris, and Cephaloscyllium umbratile) within the Carcharhiniformes and placed Squatina californica within the Squaliformes. These flaws can most likely be explained by biased and inadequate taxon sampling in different shark orders and families. The differing results between LMM and PIC analyses might be explained by failure to meet the PIC assumption of correct topology. Furthermore, continued stabilising selection (Hansen 1997) might lead to a lack of trait variability within specific lineages; therefore, any bias towards lineages where this phenomenon (or localised phylogenetic inertia) is observed will greatly bias the PIC outputs. With nearly 30 and $60 \%$ of species included in our analyses, due to limitations of availability of data, comprising a single genus (Carcharhinus) and a single order (Carcharhiniformes), respectively, there are potentially some influences of continued stabilising selection or localised phylogenetic inertia on PIC analyses of our data. These issues will not be resolvable without simultaneous increased sampling of shark diet and parasite assemblages across all shark orders.

Large-scale food web analyses have previously highlighted trophic level as an important aspect of host diet that can drive patterns of parasite richness (Lafferty et al. 2006, Chen et al. 2008). Similarly to diet breadth, we found an effect of trophic level on parasite richness, but only when data were adjusted to account for phylogenetic relationships between hosts (Fig. 5c). Trophic level was overall the third best predictor of shark tapeworm richness in this study, but there was also a notably large margin in relative importance between trophic level and the top predictor, diet breadth. It is worth noting, however, that trophic level and diet breadth had a significant interaction in the model, signifying that although these variables measure different diet aspects (trophic level reflects the position of a shark's prey in the food web, where general diet breadth does not), they are related on some level, and may both gauge how broad a shark's diet is. When considering this, one could posit that tapeworm richness in sharks is determined more by the breadth of different prey in a host species' diet, rather than by the position of these prey in food webs. A study of other marine fishes has also observed trophic level to have less impact on parasite richness compared with breadth of diet (Locke et al. 2014). Even so, there is a question of why trophic level was a key driver of parasite richness in network studies, while appearing to be of less importance here. Locke et al. (2014) offered a few plausible explanations: (1) trophic level is less relevant in the context of a fish community because it varies much less than in larger networks of species, and (2) links found between parasite richness and trophic level in other studies may reflect their association with diet breadth, meaning that diet breadth may actually be the underlying predictor of significance for parasite richness. It is also worth considering that trophic level may not appear as important in vertebrates where species at higher trophic levels have their parasite faunas restricted by more complex and effective immune responses (Benesh et al. 2014). There may be some truth to each of these explanations. In any case, further research will be necessary to fully understand the relative influences of diet breadth and trophic level on parasite richness, and considering our results, we recommend that such studies look simultaneously at both factors as predictors of parasite richness (and account for their interaction).

Prior to this study, we proposed that shark species feeding predominantly on teleost fishes would have more tapeworm species than sharks feeding mainly on other prey groups, because most currently described intermediate hosts for tapeworms are teleost fishes (Palm 2004, Jensen 2009). With our results running counter to this proposal, there is a question of why the dominant prey group of sharks does not appear to be a major determinant of tapeworm richness. One point worth noting is that crustaceans and cephalopods (the 2 other most commonly dominant prey groups of sharks) have also been described as intermediate hosts for many tapeworms, but are generally less studied for parasites than teleost fishes, meaning that they likely harbour larval stages of substantially more species of tapeworms than have been currently described (Jensen 2009). Another possibility is that the lack of importance for diet composition reflects the limitations of simplifying composition into such large taxonomic groups. Perhaps more specific diet composition involving comparisons at the species level could more accurately reflect differences in tapeworm richness. Analysing diet composition at this level would prove extremely challenging, however, since a large portion of studies on shark diets do not provide composition for individual species, genera, families, or even orders, and instead only estimate the full composition of major taxonomic groups.

Our LMM looking at predictors of the average TD of tapeworm assemblages found that no factors included in this study were good predictors of tape- 
worm TD (Table 3). This was somewhat surprising given that a few of the same variables (especially diet breadth) were considerably more important predictors of tapeworm species richness (Table 1), but as previous research has illustrated, parasite richness and average TD of parasite assemblages are sensitive to different host features (Luque et al. 2004, Heino et al. 2005, Luque \& Poulin 2008, Randhawa \& Poulin 2010). This is not to say these measures of diversity are completely different. As with the analysis of tapeworm richness, the predictors of tapeworm TD showed substantial differences when phylogenetic relationships between hosts were adjusted for using PICs, and it was similarly revealed that diet breadth, diet TD, and host size were all significant predictors of tapeworm TD after adjusting for host phylogeny (Fig. 5). It is worth noting that host phylogeny accounted for a significantly larger proportion of variation in tapeworm TD (20.15\%) compared with richness $(5.84 \%)$, and consequently, phylogeny may have had stronger confounding effects on the predictors of tapeworm TD. It is unclear why trophic level, latitudinal range, and depth mid-point were significant predictors of tapeworm richness but not tapeworm TD, and conversely, why depth range was a significant predictor of tapeworm TD but not richness. For the effect of depth range on tapeworm TD but not richness, one possible explanation is that sharks with broader depth ranges may encounter more diverse tapeworms due to crossing a wider range of habitats associated with a wider range of depths. Thus, sharks with wider depth distributions may not encounter more compatible tapeworm species than sharks with limited depth ranges, but the tapeworms they encounter could be more taxonomically diverse.

In line with our results for average tapeworm TD, we found no good predictors of variance in tapeworm TD (results in Table S3). This result indicates that the taxonomic evenness of tapeworm assemblages in sharks is unlikely to be influenced by the factors we examined. However, our dataset including variance in tapeworm TD was considerably more restricted ( $\mathrm{n}=57$ species), and this variable may be worth revisiting in the future when more shark tapeworm records are described. Variance in TD for diet proved to be more interesting given that it showed a positive association with both tapeworm richness and tapeworm TD in our PIC analyses. These results suggest that both the richness and TD of tapeworm assemblages may be generally greater for sharks with more taxonomically variable assemblages of prey in their diets. As noted above, measures of TD have rarely been used to measure diversity in the diets of different species. The results for TD herein highlight the potential insights researchers may gain by looking at measures of TD in conjunction with simpler measures of species diversity like species richness.

\section{CONCLUSIONS}

We examined a total of 91 shark species in this study, which is less than one-fifth of all described shark species known to date (Randhawa et al. 2015). However, a more complete analysis of the influence of diet on tapeworm richness would require host diet and tapeworm records to become available for many more shark species which are currently data deficient. Our study is the first to examine the influence of host diet and trophic level on parasite diversity in elasmobranchs, and to our knowledge, it is also the most comprehensive analysis of parasite diversity in sharks thus far. This gives credence to our key finding that the diet of shark species, and particularly the breadth of diet of a shark species, has important consequences for the diversity of its trophically transmitted parasites. The intricacies of this link between host diet breadth and tapeworm diversity in sharks warrant deeper exploration. Despite having records of prey for the shark species examined here, the parasites of these prey items are generally unknown, and it remains uncertain whether these prey are actually intermediate hosts contributing to shark tapeworm richness. Indeed, a number of studies have pointed out that there are major gaps in our knowledge of the life cycles of elasmobranch tapeworms (Palm 2004, Jensen \& Bullard 2010, Randhawa \& Brickle 2011, Caira \& Jensen 2014). These life cycles will need to be elucidated for a more in-depth understanding of the influence of diet breadth on tapeworm diversity.

Acknowledgements. We thank Robert Poulin and the evolutionary and ecological parasitology research group of the University of Otago for comments on an earlier version of this manuscript. We also thank Thomas Farrugia for his feedback on the science, methods, and analyses used. T.K.R. was supported by a University of Otago MSc Scholarship.

\section{LITERATURE CITED}

Ackerly DD (2000) Taxon sampling, correlated evolution, and independent contrasts. Evolution 54:1480-1492

Ackerly DD, Reich PB (1999) Convergence and correlations among leaf size and function in seed plants: a comparative test using independent contrasts. Am J Bot 86: 1272-1281 
Anderson DR (2008) Model based inference in the life sciences: a primer on evidence. Springer, New York, NY

Barton K (2013) MuMIn: Multi-model inference R package version 1.40.0. cran.r-project.org/web/packages/MuMIn (accessed 28 Nov 2017)

Benesh DP, Chubb JC, Parker GA (2014) The trophic vacuum and the evolution of complex life cycles in trophically transmitted helminths. Proc R Soc B 281:20141462

Caira JN, Healy CJ (2004) Elasmobranchs as hosts of metazoan parasites. In: Carrier JC, Musick JA, Heithaus MR (eds) Biology of sharks and their relatives. CRC Press, Boca Raton, FL, p 523-551

* Caira JN, Jensen K (2014) A digest of elasmobranch tapeworms. J Parasitol 100:373-391

Caira JN, Jensen K, Georgiev BB, Kuchta R and others (2017) An overview of tapeworms from the vertebrate bowels of the Earth. In: Caira JN, Jensen K (eds) Planetary biodiversity inventory (2008-2017): tapeworms from the vertebrate bowels of the Earth. Special Publication No. 25. The University of Kansas Natural History Museum, Lawrence, KS, p 1-20

* Chen HW, Liu WC, Davis AJ, Jordán F, Hwang MJ, Shao KT (2008) Network position of hosts in food webs and their parasite diversity. Oikos 117:1847-1855

Cortés E (1999) Standardized diet compositions and trophic levels of sharks. ICES J Mar Sci 56:707-717

Felsenstein J (1985) Phylogenies and the comparative method. Am Nat 125:1-15

Froese R, Pauly D (2017) FishBase. www.fishbase.org (accessed March 2017)

Garland T Jr, Harvey PH, Ives AR (1992) Procedures for the analysis of comparative data using phylogeneticallyindependent contrasts. Syst Biol 41:18-32

Guindon S, Gascuel O (2003) A simple, fast and accurate method to estimate large phylogenies by maximumlikelihood. Syst Biol 52:696-704

* Guindon S, Dufayard JF, Lefort V, Anisimova M, Hordijk W, Gascuel O (2010) New algorithms and methods to estimate maximum-likelihood phylogenies: assessing the performance of PhyML 3.0. Syst Biol 59:307-321

Hansen TF (1997) Stabilizing selection and the comparative analysis of adaptation. Evolution 51:1341-1351

*Harmon LJ, Weir JT, Brock CD, Glor RE, Challenger W (2008) GEIGER: investigating evolutionary radiations. Bioinformatics 24:129-131

*Heino J, Soininen J, Lappalainen J, Virtanen R (2005) The relationship between species richness and taxonomic distinctness in freshwater organisms. Limnol Oceanogr 50:978-986

Huelsenbeck JP, Ronquist F, Nielsen R, Bollback JP (2001) Bayesian inference of phylogeny and its impact on evolutionary biology. Science 294:2310-2314

Jaeger B (2016) R2glmm: computes R squared for mixed (multilevel) models. R package version 0.1.2. https://cran. r-project.org/package=r2glmm (accessed July 2018)

Jensen K (2009) Cestoda (Platyhelminthes) of the Gulf of Mexico. In: Felder DL, Camp DK (eds) Gulf of Mexico origin, waters, and biota: biodiversity. Texas A\&M University Press, College Station, TX, p 487-522

Jensen K, Bullard SA (2010) Characterization of a diversity of tetraphyllidean and rhinebothriidean cestode larval types, with comments on host associations and life cycles. Int J Parasitol 40:889-910

Kamiya T, O'Dwyer K, Nakagawa S, Poulin R (2014) What determines species richness of parasitic organisms? A meta-analysis across animal, plant and fungal hosts. Biol Rev Camb Philos Soc 89:123-134

Lafferty KD, Dobson AP, Kuris AM (2006) Parasites dominate food web links. Proc Natl Acad Sci USA 103: 11211-11216

* Lefort V, Longueville JE, Gascuel O (2017) SMS: Smart Model Selection in PhyML. Mol Biol Evol 34:2422-2424

ㄴocke SA, Marcogliese DJ, Valtonen ET (2014) Vulnerability and diet breadth predict larval and adult parasite diversity in fish of the Bothnian Bay. Oecologia 174:253-262

KLuque JL, Poulin R (2007) Metazoan parasite species richness in Neotropical fishes: hotspots and the geography of biodiversity. Parasitology 134:865-878

* Luque JL, Poulin R (2008) Linking ecology with parasite diversity in Neotropical fishes. J Fish Biol 72:189-204

Luque JL, Mouillot D, Poulin R (2004) Parasite biodiversity and its determinants in coastal marine teleost fishes of Brazil. Parasitology 128:671-682

Maddison DR, Maddison WP (2005) MacClade 4, version 4.07. Sinauer Associates Inc, Sunderland, MA

Maddison WP, Maddison DR (2017) Mesquite: a modular system for evolutionary analysis. Version 3.31. www. mesquiteproject.org (accessed 28 Nov 2017)

Midford PE, Garland T Jr, Maddison WP (2011) 'PDAP: PDTREE' package for Mesquite, Version 1.16. http:// mesquiteproject.org/pdap_mesquite/ (accessed 28 Nov 2017)

Morand S, Cribb TH, Kulbicki M, Rigby MC and others (2000) Endoparasite species richness of New Caledonian butterfly fishes: host density and diet matter. Parasitology 121:65-73

Naylor GJP, Caira JN, Jensen K, Rosana KAM, White WT, Last PR (2012) A DNA sequence-based approach to the identification of shark and ray species and its implications for global elasmobranch diversity and parasitology. Bull Am Mus Nat Hist 367:1-262

Palm HW (2004) The Trypanorhyncha Diesing, 1863. PKSPL-IPB Press, Bogor

* Palm HW, Caira JN (2008) Host specificity of adult versus larval cestodes of the elasmobranch tapeworm order Trypanorhyncha. Int J Parasitol 38:381-388

* Posada D (2008) jModelTest: phylogenetic model averaging. Mol Biol Evol 25:1253-1256

Foulin R (2004) Macroecological patterns of species richness in parasite assemblages. Basic Appl Ecol 5:423-434

Foulin R, Leung TLF (2011) Body size, trophic level, and the use of fish as transmission routes by parasites. Oecologia 166:731-738

* Poulin R, Guilhaumon F, Randhawa HS, Luque JL, Mouillot D (2011) Identifying hotspots of parasite diversity from species-area relationships: host phylogeny versus host ecology. Oikos 120:740-747

R Development Core Team (2012) R: a language and environment for statistical computing, Version 2.15.0. www.rproject.org (accessed November 2017)

* Randall PJ, Myers AA (2001) Effects of resource matrix, gut region analysed and sample size on diet statistics in Coexisting species of flatfish. J Mar Biol Assoc UK 81: 1041-1048

Kandhawa HS, Brickle P (2011) Larval parasite gene sequence data reveal cryptic trophic links in life cycles of porbeagle shark tapeworms. Mar Ecol Prog Ser 431: 215-222

Randhawa HS, Poulin R (2010) Determinants of tapeworm species richness in elasmobranch fishes: untangling envi- 
ronmental and phylogenetic influences. Ecography 33: 866-877

Randhawa HS, Poulin R, Krkošek M (2015) Increasing rate of species discovery in sharks coincides with sharp population declines: implications for biodiversity. Ecography 38:96-107

Ronquist F, Huelsenbeck J, Teslenko M (2011) MrBayes version 3.2 manual: tutorials and model summaries. http:// mrbayes.sourceforge.net/mb3.2_manual.pdf (accessed 28 Nov 2017)

Stringell TB, Clerveaux WV, Godley BJ, Kent FEA and others (2016) Taxonomic distinctness in the diet of two sympatric marine turtle species. Mar Ecol 37:1036-1049

Vitone ND, Altizer S, Nunn CL (2004) Body size, diet and sociality influence the species richness of parasitic worms in anthropoid primates. Evol Ecol Res 6:183-199

Editorial responsibility: Inna Sokolova, Rostock, Germany
Von Euler F, Svensson S (2001) Taxonomic distinctness and species richness as measures of functional structure in bird assemblages. Oecologia 129:304-311

*Walther BA, Cotgreave P, Price RD, Gregory RD, Clayton DH (1995) Sampling effort and parasite species richness. Parasitol Today 11:306-310

Warwick RM, Clarke KR (2001) Practical measures of marine biodiversity based on relatedness of species. Oceanogr Mar Biol Annu Rev 39:207-231

Williams HH, Jones A (1994) Parasitic worms of fish. Taylor \& Francis, London

*Winter M, Devictor V, Schweiger O (2013) Phylogenetic diversity and nature conservation: Where are we? Trends Ecol Evol 28:199-204

WoRMS Editorial Board (2017) World Register of Marine Species. www.marinespecies.org (accessed May 2017)

Submitted: January 24, 2018; Accepted: September 5, 2018 Proofs received from author(s): October 3, 2018 